TUBERCULOSIS

\title{
Ethnic differences in selective neonatal BCG immunisation: white British children miss out
}

\author{
R Srinivasan, L Menon, P Stevens, I Campbell, M Alfaham
}

See end of article for authors' affiliations

\section{..................... \\ Correspondence to:} Dr R Srinivasan,

Department of Child Health, Llandough Hospital, Cardiff CF64

2XX, UK; ramsriniv@

doctors.org.uk

Received 16 October 2004 Accepted

21 November 2005

Published Online First

29 December 2005
Background: Tuberculosis (TB) is a re-emerging problem, especially in the larger cities of Western Europe. Selective neonatal BCG vaccination is recommended for infants at risk of TB in the UK. Neonatal BCG is safe and effective, with an overall protective value of $75 \%$. This study aimed to assess BCG rates among at risk infants in Cardiff and the Vale of Glamorgan, South Wales in the year 2003.

Methods: A cohort of infants at risk for TB was identified from demographic data stored on a computerised maternity activity database. A manual search of immunisation records determined overall rates and the rates for infants belonging to various ethnic groups.

Results: Of 5308 infants born in 2003, 514 (9.6\%) were at risk of TB; 423 (82.2\%) of these infants were referred postnatally for BCG vaccination and 391 received it. Twenty six of the 41 at risk white British infants missed having a BCG vaccination compared with 47 of 288 Asian infants and seven of 39 black African babies. The rate of BCG vaccination among white British infants was $36.5 \%$ compared with $83.6 \%$ for Asian infants from the Indian subcontinent $\left(\chi^{2}=7.25, p<0.01\right)$ and $82 \%$ for black African infants $\left(\chi^{2}=4.48, p<0.05\right)$.

Conclusions: The overall BCG rate among at risk infants in Cardiff was $76 \%$ during the study period. The vaccination rate was poor among white British infants compared with other ethnic groups. Enhanced awareness of health professionals to recognise the need for vaccinating certain white children at risk of TB is essential to improve BCG coverage in an increasingly multiethnic population.
$\mathrm{S}$ ince the beginning of the 19th century, notification rates for tuberculosis (TB) have been on the decline owing to improvements in living conditions, nutrition, introduction of anti- tuberculosis chemotherapy, and Bacille Calmette-Guerin (BCG) immunisation. However, TB is now re-emerging, especially in the larger cities of Western Europe. ${ }^{1}$ Increasing levels of deprivation in the inner city areas and immigration of families from countries with a high prevalence of $\mathrm{TB}$ are probably the important factors responsible for this trend. ${ }^{2}$

In the UK, notifications of TB declined tenfold between 1948 and 1987. However, the steady increase since then is a matter of concern. ${ }^{3}$ In a survey conducted by the Public Health Laboratory Service in England and Wales the annual rate for newly notified cases was 10.93 per 100000 population in 1998. This represents an increase of $11 \%$ since the 1993 survey and $21 \%$ since $1988 .{ }^{4}$ Childhood TB notification rates in England and Wales decreased at an average rate of $7.2 \%$ each year from 1978 to 1988 , but there has been a steady increase in notification rates ever since. ${ }^{5}$ Surveillance of childhood TB from September 1996 to December 1999 conducted through the Welsh Paediatric Surveillance System reported 38 cases of TB and 59 children on chemoprophylaxis. The majority of cases were noted to be in the white population and most had not received BCG vaccination. ${ }^{6}$ This was consistent with a previous study conducted in Wales. ${ }^{7}$ Although South Wales is a low prevalence area, TB in childhood is increasing and preventive measures are clearly important. We therefore undertook this study to assess the coverage of a selective neonatal BCG immunisation programme aimed at vaccinating infants at risk for TB.

\section{METHODS}

We conducted a review of the selective neonatal BCG immunisation programme within the Cardiff and Vale NHS
Trust which serves the population of Cardiff City and the Vale of Glamorgan, South Wales. Local guidelines exist for the identification of infants at risk for TB who should be referred for BCG vaccination (box 1). ${ }^{3-11}$

There is a single tick box for TB risk in the antenatal clerking sheet. The information needed to categorise risk is assessed at the pregnancy booking interview. All midwifery staff are provided with the criteria for identifying need for selective neonatal BCG vaccination. However, sensitive information relating to drug abuse and travelling status may be difficult to elicit, and details are not explicit in these forms apart from the need to vaccinate these infants (in some cases this information may be available only postnatally). These data are held on a computerised maternity database maintained by the Cardiff birth survey. However, referral to

\section{Box 1 Selective neonatal BCG immunisation:} criteria for referral (TB Control Service, Cardiff and Vale NHS Trust)

- Parents originating from India, Pakistan, Bangladesh, Asia, Africa, Eastern Europe, Central \& South America, Caribbean and Middle East, even if UK born.

- Parents who are asylum seekers/refugees from any country.

- Parent/close living relative with active TB or a past history of TB.

- Parent/close living relative who is a close contact of pulmonary TB and has been asked to attend for screening.

- Those from travelling families.

- History of parental drug abuse. 


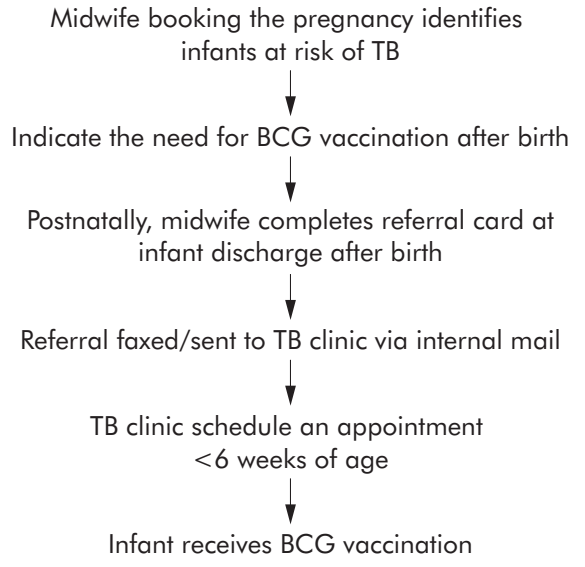

Figure 1 Process of BCG vaccination.

the BCG clinic takes place postnatally by midwifery staff following birth of the infant. If missed at discharge, infants can also be referred by the health visitor, general practitioner, or other healthcare professionals to be vaccinated by the team of two TB clinical nurse specialists who run the BCG vaccination service. A manual documentation of referrals received, together with the original or faxed referral forms, are kept on file. The aim is to vaccinate infants within 6 weeks of birth. Details of vaccination are recorded on BCG vaccination cards. Infants not immunised by 3 months of age are tuberculin tested and, if found to be tuberculin negative, they are vaccinated. Infants found to be tuberculin positive are referred to local paediatric services for investigations and appropriate management. The vaccination process is summarised in fig 1 .

Demographic data on pregnancies delivered between 1 January and 31 December 2003 were available from the maternity database. A list of infants at risk for TB was generated. Details of postnatal referral and vaccination outcome were retrieved from the manual records kept by the TB team. The efficacy of the vaccination process was determined by calculating the percentage of infants at risk who received BCG vaccination. In addition, vaccination rates for infants belonging to various ethnic groups were computed and the differences analysed using the $\chi^{2}$ test.

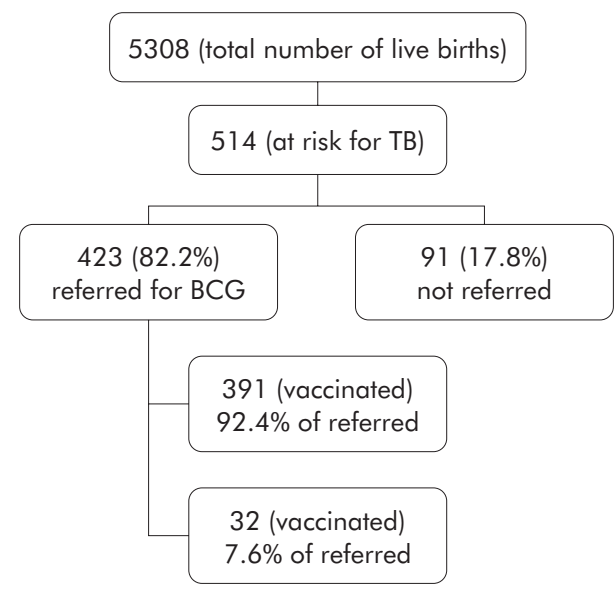

Figure 2 Uptake of neonatal BCG vaccination in Cardiff and Vale of Glamorgan, 2003.

\section{RESULTS}

Of the 5308 live births recorded in the year 2003, a total of 514 infants $(9.6 \%)$ were identified to be at risk for TB from computerised records based on antenatal information. Referrals for vaccination (done postnatally) were received for only 423 infants (82.2\%). By l March 2004, 391 infants had received vaccination $(76.0 \%$ of at risk infants or $92.4 \%$ of those referred). Nineteen infants (3.6\%) had failed to attend repeated appointments. Five infants $(0.9 \%)$ could not be traced. Eight infants (1.5\%) were awaiting vaccination in the subsequent weeks. The pattern of BCG vaccination is depicted in fig 2 .

The largest group of infants at risk (56\%) were born to Asian parents originating from the Indian subcontinent (Indian, Pakistani and Bangladeshi), and $83.6 \%$ of these infants received BCG vaccination. The vaccination rate among infants born to Black African parents was much the same $(82 \%)$. Of 41 white British infants, only $15(36.5 \%)$ received BCG vaccination; 26 (63.5\%) were not referred and one infant was not brought to the BCG clinic despite repeated appointments. The vaccination rate was $71 \%$ among the 146 infants from the remaining ethnic groups which included Chinese $(n=16)$, other black $(n=7)$, other white $(n=6)$, other Asian $(n=31)$, mixed $(n=52)$, unclassified $(n=28)$, and not stated $(\mathrm{n}=6)$, as shown in table 1 .

\section{DISCUSSION}

BCG vaccination is an effective preventive measure against TB, although estimates of its efficacy vary enormously between different studies. In a meta-analysis of four randomised control trials addressing the efficacy of neonatal BCG, an overall protective rate of $74 \%$ was reported. It was higher $(78 \%)$ for miliary TB but slightly lower $(64 \%)$ against TB meningitis. ${ }^{12}$ The recommendations for BCG vaccination in infants vary between the countries of Western Europe, and even between cities of the same country in a few instances. In France, Finland, and Portugal BCG vaccination in infancy is universal, while only high risk groups are targeted in Belgium, Greece, and the UK. Infants belonging to high risk groups receive the vaccine in Milan (Italy) and Cork (Ireland), while those born in Rome and Dublin do not. ${ }^{1}$

The Department of Health for England, the Welsh and Scottish Offices, ${ }^{3}$ and the British Thoracic Society ${ }^{8}$ currently advise targeting infants belonging to high risk groups for vaccination. Our study found an overall BCG vaccination rate of $76 \%$. Ahmed and Hicks from Bristol showed a rate of $13.3 \%{ }^{13}$ while Gill and Scott reported a rate of $6 \%$ in Bolton. ${ }^{14}$ Both these studies demonstrated improvements to over $80 \%$ after intensive training of midwifery and health visiting personnel. Wyllie and Eastham found a $42 \%$ rate in Middlesborough, ${ }^{15}$ while Deshpande reported a BCG vaccination rate of $51 \%$ in at risk infants in Shropshire. ${ }^{16}$ It is important to note that none of the previous studies

Table 1 Vaccination uptake status according to ethnicity

\begin{tabular}{llll}
\hline Ethnic group & $\begin{array}{l}\text { At risk } \\
\text { (frequency) }\end{array}$ & $\begin{array}{l}\text { Vaccinated } \\
\text { (frequency) }\end{array}$ & $\begin{array}{l}\text { Uptake } \\
\text { (\%) }\end{array}$ \\
\hline Asian: Pakistani & 96 & 81 & 84.3 \\
Asian: Indian & 124 & 102 & 82.2 \\
Asian: Bangladeshi & 68 & 58 & 85.3 \\
Black African & 39 & 32 & 82.0 \\
White British & 41 & 15 & 36.5 \\
Other ethnic groups & 146 & 103 & 70.5 \\
Total & 514 & 391 & 76.0 \\
\hline
\end{tabular}

There were significant differences in the vaccination rates for white British infants and those for Asian and black African infants (Asian $83.6 \% \mathrm{v}$ white British 36.5\%: $\chi^{2}=7.25, \mathrm{p}<0.01$; black African $82 \% v$ white British 36.5\%: $\left.\chi^{2}=4.48, \mathrm{p}<0.05\right)$. 
emphasised the low BCG uptake among white British infants at risk. The difference in the percentage rates between the different ethnic groups in our study is a concern-only a third of white British infants received BCG vaccination compared with over $80 \%$ for Asian and black African infants. White British infants at risk of TB would be predominantly referred under three categories: family history of TB, parental drug abuse, or belonging to travelling families. The low uptake among white British infants may be attributed to the failure of healthcare professionals to appreciate that, among this ethnic grouping, there are certain infants at increased risk of TB. A presumption of no risk for TB just because an infant is of white ethnicity is seemingly the reason for this.

Lack of computerised postnatal records linked to antenatal data and lack of communication among healthcare professionals are possible shortcomings. Practical difficulties are also inherent in the implementation of such a selective immunisation programme where the definition of what is considered to be "high risk" is not always straightforward and some children in need of immunisation may be missed primarily due to a lack of this awareness. Lack of written communication from those assessing risk initially, shortages of BCG supply, travelling families, and changes of infant surnames add to the difficulty of ensuring adequate BCG uptake.

The results of this study have been presented to the midwifery teams and at Paediatric and Obstetric departmental meetings. Documentation of ethnicity on BCG vaccination cards and recording of the indication for referral have been implemented. Increased awareness of perinatal staff regarding the high risk categories for TB and heightened perception of the vulnerability of a certain group of white infants are the keys to improving coverage. The results of this study have a wider applicability-namely, the importance of recognising that we live in an increasingly multiethnic European society which brings forth certain common healthcare provision issues.

\section{ACKNOWLEDGEMENTS}

The authors thank Mrs Gwyneth Ratcliffe of the Cardiff birth survey for access to computerised antenatal records and demographic information.

\section{Authors' affiliations}

R Srinivasan, L Menon, M Alfaham, Department of Child Health, Llandough Hospital, Cardiff, UK

P Stevens, TB Control Service, Llandough Hospital, Cardiff, UK

I Campbell, Department of Respiratory Medicine, Llandough Hospital,

Cardiff, UK

Conflict of interest and sources of funding: none.

\section{REFERENCES}

1 Hayward AC, Darton T, Van-Tam JN, et al. Epidemiology and control of tuberculosis in Western European cities. Int J Tuberc Lung Dis, 7:751-7.

2 Bannon MJ. BCG and tuberculosis. Arch Dis Child 1999:80:80-3.

3 Department of Health, Welsh Office, Scottish Office Department of Health, DHSS (Northern Ireland). Immunisation against infectious disease, London, 1996.

4 Rose AMC, Watson JM, Graham C, et al. Tuberculosis at the end of the 20th century in England and Wales: results of a national survey in 1998. Thorax 2001;56:173-9.

5 Medical Research Council Cardiothoracic Epidemiology Group. Tuberculosis in children: a national survey of notifications in England and Wales in 1988. Arch Dis Child 1994;70:497-500.

6 Sastry J, Alfaham M, Evans $M$. Surveillance of childhood tuberculosis through the Welsh Paediatric Surveillance System (WPSS). Thorax 2000;55(Suppl 3):A56.

7 Mathew V, Alfaham M, Evans MR, et al. Management of tuberculosis in Wales: 1986-92. Arch Dis Child 1998;78:349-53.

8 British Thoracic Society. Control and prevention of tuberculosis in the United Kingdom: code of practice 2000. Thorax 2000;55:887-901.

9 Callister ME, Barringer J, Thanabalasingam ST, et al. Pulmonary tuberculosis among political asylum seekers at Heathrow Airport, London, 1995-9. Thorax 2002;57:152-6.

10 Drobniewski FA, Balabanova YM, Ruddy MC, et al. Tuberculosis, HIV seroprevalence and intravenous drug abuse in prisoners. Eur Respir $J$ 2005;26:298-304.

11 Garcia Sanchez I, Perez de Oteyza C, Gilsanz Fernandez C. Tuberculosis epidemiological study in a third level hospital during 2001. An Med Interna 2005;22:222-6.

12 Colditz GA, Berkey CS, Mosteller F, et al. The efficacy of Bacillus CalmetteGuerin vaccination of newborns and infants in the prevention of tuberculosis: meta-analyses of the published literature. Pediatrics 1995;96:29-35.

13 Ahmed S, Hicks NR, Stanwell-Smith R. Policy and practice: an audit of neonatal BCG immunization in Avon. J Public Health Med 1992;14:389-92.

14 Gill J, Scott J. Improving the uptake of selective neonatal BCG immunisation. Commun Dis Public Health 1998;1:281-2.

15 Eastham KM, Wyllie J. A study of neonatal BCG immunization within an acute hospital trust. J Public Health Med 2001;23:335-8.

16 Deshpande SA. Ethnic differences in the rates of BCG vaccination. Arch Dis Child 2004;89:48-9. 